\title{
Perfil de Risco de Reestenose em Pacientes Submetidos a Implante de Stents Coronarianos Convencionais
}

\author{
Alexandre Schaan de Quadros ${ }^{1}$, Carlos A. M. Gottschall ${ }^{1}$, Fabiane Diemer ${ }^{1}$, Dayane Diehl', \\ Rogério Sarmento-Leite ${ }^{1}$, Ana Paula da Rosa Rodrigues', Mateus D. Vizzotto', Fernanda Camozzatto', \\ João M. P. Martins ${ }^{1}$, La Hore Corrêa Rodrigues Junior ${ }^{1}$, Giana Sassi ${ }^{1}$, Thais B. Modkovski ${ }^{1}$
}

\section{RESUMO}

Introdução: O perfil de risco de reestenose de populações tratadas com implante de stents coronarianos em nosso meio não é conhecido. Essa informação tem importância na decisão de incorporar uma estratégia seletiva de implante de stents farmacológicos pelo sistema público de saúde. Objetivos: Avaliar o risco de reestenose antes do procedimento de uma população de pacientes tratados com stents coronarianos convencionais. Método: Estudo observacional de corte transversal, com 4.482 pacientes tratados com 5.336 stents, no período de janeiro de 2000 a dezembro de 2007. O risco de reestenose foi avaliado conforme escore previamente validado, com pontuação de 0 a 5 conforme a presença de diabetes melito ( 1 ponto), o diâmetro de referência do vaso tratado $(<3 \mathrm{~mm}=2$ pontos, $3-3,5 \mathrm{~mm}=1$, e $>3,5 \mathrm{~mm}=0$ ) e a extensão da lesão ( $20 \mathrm{~mm}=2$ pontos, $10-20 \mathrm{~mm}=1$, e $<10 \mathrm{~mm}=0$ ). Resultados: A média de idade foi de 60,6 \pm 10,6 anos e $32 \%$ dos pacientes eram do sexo feminino. O diâmetro de referência do vaso tratado foi de $3,10 \pm 0,51 \mathrm{~mm}$, a extensão da lesão foi de 13,2 $\pm 5,9 \mathrm{~mm}$ e $20 \%$ dos pacientes apresentavam diabetes melito. A distribuição dos pacientes conforme os pontos no escore de reestenose foi a seguinte: escore $0=4 \%$ dos pacientes; escore $1=22 \%$; $2=34 \% ; 3=29 \% ; 4=9 \%$; e escore $5=1 \%$ dos pacientes. Conclusão: A maioria dos pacientes apresentou risco de reestenose baixo ou intermediário. A adoção de stents farmacológicos somente naqueles com alto risco de reestenose representaria seu uso em no máximo 20\% dos procedimentos realizados atualmente.

DESCRITORES: Contenedores. Reestenose coronária. Angioplastia.

\section{SUMMARY}

Restenosis Risk Profile of Patients Submitted to Coronary Bare-Metal Stent Implantation

Background: The restenosis risk of patient populations treated with coronary stent implantation is not well studied. This information has a potential impact on the decision of incorporating a selective strategy of drug-eluting stent implantation by the public health system. Our objective was to evaluate the restenosis risk of a population of patients (pts) treated with bare-metal stents. Methods: Observational study with 4,482 pts treated with 5,336 stents, between January 2000 and December 2007. The restenosis risk was assessed according to a previous validated risk score. Points in the score ranged from 0 to 5 according to diabetes mellitus ( 1 point), reference vessel diameter $(<3 \mathrm{~mm}=2$ points, $3-3.5 \mathrm{~mm}=1$, and $>3.5 \mathrm{~mm}=0)$, and the lesion length $(>20 \mathrm{~mm}=2$ points, $10-20 \mathrm{~mm}=1$, and $<10 \mathrm{~mm}=0$ ). Results: The mean age was $60.6 \pm 10.6$ years of age, and $32 \%$ were female. The mean reference vessel diameter was $3.10 \mathrm{~mm} \pm 0.51 \mathrm{~mm}$, the lesion length was $13.2 \mathrm{~mm} \pm 5.9 \mathrm{~mm}$, and $20 \%$ of the pts were diabetics. The distribution of pts according to points in the risk score was the following: score $0=4 \%$ of the pts; score $1=22 \%$; $2=34 \% ; 3=29 \% ; 4=9 \%$; and score $5=1 \%$ of the pts. Conclusions: The majority of pts presented low or intermediate restenosis risk. The adoption of a selective strategy of drug-eluting stent implantation only in those at higher restenosis risk would represent its use in no more than $20 \%$ of the procedures.

DESCRIPTORS: Stents. Coronary restenosis. Angioplasty.

\footnotetext{
1 Instituto de Cardiologia, Fundação Universitária de Cardiologia Porto Alegre, RS.

Correspondência: Alexandre Quadros. Unidade de Pesquisa do IC/ FUC. Av. Princesa Isabel, 370 - Santana - Porto Alegre, RS CEP 90620-001 - Fax: (51) 3219-2802/ramais 22-23-24

E-mail: aquadros@cardiol.br

Recebido em: 9/5/2008 • Aceito em: 15/5/2008

Suporte Financeiro: CNPq, FAPERGS
} 
0 s stents farmacológicos estão associados a taxas significativamente menores de reestenose que os stents convencionais ${ }^{1-3}$. Essa é uma característica importante desses dispositivos, já que a reestenose está associada a piora da qualidade de vida e a síndromes coronarianas agudas em alguns pacientes ${ }^{4-6}$. Além disso, estudos recentes têm demonstrado que os stents farmacológicos não estão associados a risco aumentado de morte e infarto do miocárdio (IAM) na maioria das situações clínicas e angiográficas e quando terapia antiplaquetária dupla adequada é instituída e mantida ${ }^{7-10}$. Nesse contexto, questões financeiras parecem ser o maior problema para que os stents farmacológicos sejam utilizados em maior número de pacientes, já que são significativamente mais caros que os stents convencionais. Por outro lado, mesmo com custos iniciais maiores, análises econômicas têm demonstrado que os stents farmacológicos podem ser custo-efetivos ou mesmo economizar recursos em pacientes com alto risco de reestenose, por diminuir significativamente a necessidade de novos procedimentos de revascularização ${ }^{11,12}$.

O risco de reestenose de populações de pacientes tratados com stents coronarianos no Brasil não é bem estudado. Essa informação tem importante impacto potencial na decisão de adotar uma estratégia seletiva de implante de stents farmacológicos pelo sistema público de saúde. Nosso grupo recentemente desenvolveu e validou um escore de risco para avaliar o risco de reestenose após implante de stents coronarianos convencionais, baseado em variáveis obtidas antes do procedimento ${ }^{13,14}$. O objetivo desse estudo é a avaliação do risco de reestenose em população de pacientes tratados em um centro de referência para Cardiologia Intervencionista.

\section{MÉTODO}

\section{Pacientes}

Todos os pacientes submetidos a intervenção coronariana percutânea (ICP) com implante de stent para doença arterial coronariana sintomática em nossa instituição foram considerados para inclusão no estudo. Os pacientes foram tratados entre janeiro de 2000 e dezembro de 2007. As características clínicas, angiográficas e do procedimento em relação à população em estudo foram registradas prospectivamente e armazenadas em banco de dados computadorizado. Foram excluídos aqueles pacientes tratados com stents farmacológicos.

O estudo foi aprovado pelo Comitê de Ética em Pesquisa da instituição.

\section{Procedimento de implante de stent}

Todos os pacientes estavam recebendo inibidores plaquetários no momento da intervenção: aspirina (100200 mg por dia) e tienopiridínicos (clopidogrel 75 mg por dia ou ticlopidina $250 \mathrm{mg}$ duas vezes por dia). Nos casos urgentes, as drogas foram administradas antes ou logo após o procedimento, e as doses em bolus de clopidogrel foram de $300 \mathrm{mg}$. Bolus de heparina foram administrados durante o procedimento para manter tempo de coagulação ativado > 300 segundos (ou 200300 segundos naqueles recebendo inibidores de glicoproteína IIb/IIla). As lesões foram tratadas com as técnicas padrão de $I C P^{15}$. Os demais aspectos técnicos, como tipo e número de stents implantados, uso de outros dispositivos e farmacologia adjunta, ficaram a cargo das decisões dos médicos operadores.

\section{Análise angiográfica}

Todas as análises angiográficas foram realizadas em pelo menos duas projeções ortogonais, por operadores experientes, por meio de angiografia quantitativa com sistemas digitais previamente validados (Siemens Axiom Artis - Munique, Alemanha). Nitroglicerina intracoronariana foi administrada rotineiramente em dose de 100-200 $\mu \mathrm{g}$ antes das mensurações. O diâmetro do vaso-alvo foi definido como a média do diâmetro dos segmentos proximal e distal à lesão, e a gravidade da estenose foi medida em duas projeções ortogonais. O comprimento da lesão foi medido "ombro a ombro", sendo as lesões longas consideradas únicas quando existia um segmento normal $<10 \mathrm{~mm}$ entre elas.

\section{Escore de risco}

O escore utilizado para avaliar o risco de reestenose nesse estudo foi desenvolvido em uma coorte prospectiva de 848 pacientes e, subseqüentemente, validado em outra amostra independente de 491 pacientes, conforme previamente descrito ${ }^{13,14}$. No estudo de desenvolvimento, os preditores de revascularização do vaso-alvo (RVA) em um ano foram identificados por análise multivariada, e o teste de HosmerLemeshow goodness-of-fit foi utilizado para identificar o modelo com melhor calibração. A presença de diabetes melito, o diâmetro de referência do vaso-alvo e o comprimento da lesão permaneceram no modelo final, e os pontos no escore foram atribuídos conforme os riscos relativos de cada variável:

1. diabetes melito: presente $=1$ ponto e ausente $=0$ pontos;

2. diâmetro de referência do vaso: $<3,0 \mathrm{~mm}=2$ pontos, 3,0-3,5 $\mathrm{mm}=1$ ponto e $>3,5 \mathrm{~mm}=0$ pontos;

3. comprimento da lesão: $>20 \mathrm{~mm}=2$ pontos, $10-20 \mathrm{~mm}=1$ ponto $\mathrm{e}<10 \mathrm{~mm}=0$ pontos.

De acordo com essa metodologia, o escore variou de 0 a 5 pontos. Pacientes com escores de 4 ou 5 foram incluídos na mesma categoria, já que poucos pacientes apresentaram escore de 5/5.

Na Figura 1, podemos observar a variação nas taxas de RVA em um ano, conforme os pontos no escore, sendo a associação entre ambos estatisticamente significativa, tanto na coorte de desenvolvimento $(p<0,001)$ como na de validação ( $p=0,008)$. 


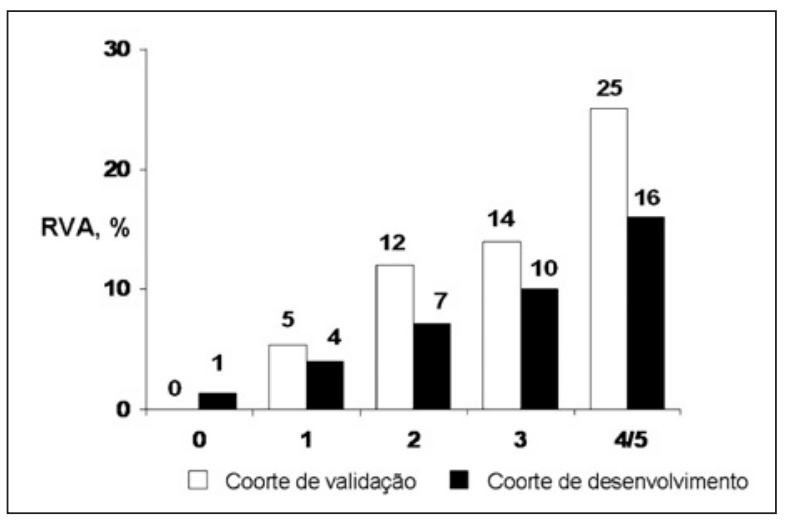

Figura 1 - Taxas de revascularização do vaso-alvo em um ano nas coortes de desenvolvimento e validação do escore de risco. RVA = revascularização do vaso-alvo.

\section{Análise estatística}

Os dados foram armazenados em banco de dados Access e analisados em programa estatístico SPSS for Windows 11.0.

Nesse estudo, as variáveis categóricas foram descritas como percentis e as variáveis contínuas, conforme sua média \pm desvio padrão.

\section{RESULTADOS}

No período do estudo, foram incluídos 4.482 pacientes tratados com 5.336 stents coronarianos convencionais. Em relação às características clínicas dos pacientes, a média de idade foi de 60,64 \pm 10,65 anos e $32 \%$ eram mulheres (Tabela 1). Quanto aos fatores de risco para cardiopatia isquêmica, hipertensão foi observada em $75 \%$ dos pacientes, diabetes melito em $20 \%$, tabagismo em 47\% e dislipidemia em 56\%. Em relação à história médica pregressa, $9 \%$ já haviam realizado uma ICP, 20\% tinham história de IAM, e $10 \%$ haviam sido submetidos a revascularização cirúrgica. A apresentação clínica no momento do implante do stent foi de angina estável em $42 \%$, angina instável em $48 \%$ e IAM em $10 \%$.

As características angiográficas dos 5.336 procedimentos de implante de stents encontram-se na Tabela 2. Os procedimentos foram mais freqüentemente realizados em lesões nas artérias descendente anterior e direita (41\% e 30\% dos casos, respectivamente), $17 \%$ dos quais foram realizados em lesões da artéria circunflexa e somente 4\% em pontes de safena. Quanto aos dados da análise por angiografia quantitativa, observou-se que a média do diâmetro de referência do vaso tratado foi de 3,10 $\pm 0,51 \mathrm{~mm}$, a média do comprimento da lesão foi de 13,2 $\pm 5,9 \mathrm{~mm}$, e o porcentual da estenose antes do implante foi de 87,05 \pm $11,59 \%$. Quanto à extensão da lesão, $8 \%$ apresentavam lesões > $20 \mathrm{~mm}$, e em relação ao diâmetro de referência, $28 \%$ apresentavam vasos $<3 \mathrm{~mm}$. Os stents
TABELA 1

Características clínicas dos pacientes

\begin{tabular}{lc}
\hline & Número de pacientes = \\
& $\mathbf{4 . 4 8 2}$ \\
\hline Idade, anos & $60,64 \pm 10,65$ \\
Mulheres & $32 \%$ \\
Fatores de risco & \\
Hipertensão & $75 \%$ \\
Diabetes melito & $20 \%$ \\
Tabagismo & $47 \%$ \\
Dislipidemia & $56 \%$ \\
História médica & \\
Intervenção coronariana & \\
percutânea & $9 \%$ \\
Infarto agudo do miocárdio & $20 \%$ \\
Cirurgia de revascularização & \\
do miocárdio & $10 \%$ \\
Apresentação clínica & \\
Angina estável & $42 \%$ \\
Angina instável & $48 \%$ \\
Infarto agudo do miocárdio & $10 \%$ \\
\hline
\end{tabular}

TABELA 2

Características angiográficas dos procedimentos de implante de stents

Número de stents $=$ 5.336

\begin{tabular}{lc}
\hline Vaso tratado & \\
Artéria descendente anterior & $41 \%$ \\
Artéria circunflexa & $17 \%$ \\
Artéria direita & $30 \%$ \\
Ponte de safena & $4 \%$ \\
Multiarteriais & $29 \%$ \\
Diâmetro de referência do vaso & $3,10 \pm 0,51 \mathrm{~mm}$ \\
Comprimento da lesão & $13,2 \pm 5,9 \mathrm{~mm}$ \\
Diâmetro do stent & $3,15 \pm 1,02 \mathrm{~mm}$ \\
Comprimento do stent & $16,05 \pm 5,26 \mathrm{~mm}$ \\
Pressão de implante & $13,92 \pm 2,48 \mathrm{~atm}$ \\
\% de estenose & $87,05 \pm 11,59 \%$ \\
Diâmetro luminal mínimo & $0,46 \pm 0,37 \mathrm{~mm}$ \\
Diâmetro luminal final & $3,16 \pm 0,61 \mathrm{~mm}$ \\
\hline
\end{tabular}

implantados apresentaram diâmetro médio de 3,15 \pm 1,02 mm, comprimento de 16,05 $\pm 5,26 \mathrm{~mm}$, e pressão de implante de 13,92 $\pm 2,48$ atm. O diâmetro luminal mínimo antes do implante foi de 0,46 $\pm 0,37 \mathrm{~mm}$, aumentando para 3,16 \pm 0,61 $\mathrm{mm}$ após o procedimento.

A Figura 2 demonstra a distribuição dos pacientes conforme os pontos no escore de risco: escore $0=4 \%$ dos 
pacientes; escore $1=22 \% ; 2=34 \% ; 3=29 \% ; 4=9 \%$; e escore $5=1 \%$ dos pacientes. A observação dessa figura sugere distribuição próxima à curva normal.

Na Figura 3, esses resultados estão apresentados conforme três categorias de risco. Foram considerados pacientes de baixo risco para reestenose aqueles com escores iguais a 0 ou 1 , médio risco aqueles com escores de 2 ou 3, e alto risco os pacientes com escores de 4 ou 5. Assim, observamos que a maioria dos pacientes apresentava risco intermediário (64\%), $26 \%$ apresentavam risco médio, e somente $10 \%$ apresentavam alto risco.

\section{DISCUSSÃO}

Nesse estudo, analisamos as características clínicas e angiográficas de uma grande população de pacientes tratados com stents coronarianos convencionais em um hospital de referência para Cardiologia Intervencionista, sendo o perfil de risco de reestenose estimado por um escore de risco previamente validado. Os resultados da análise demonstram que a maioria dos pacientes apresenta risco baixo ou intermedi-

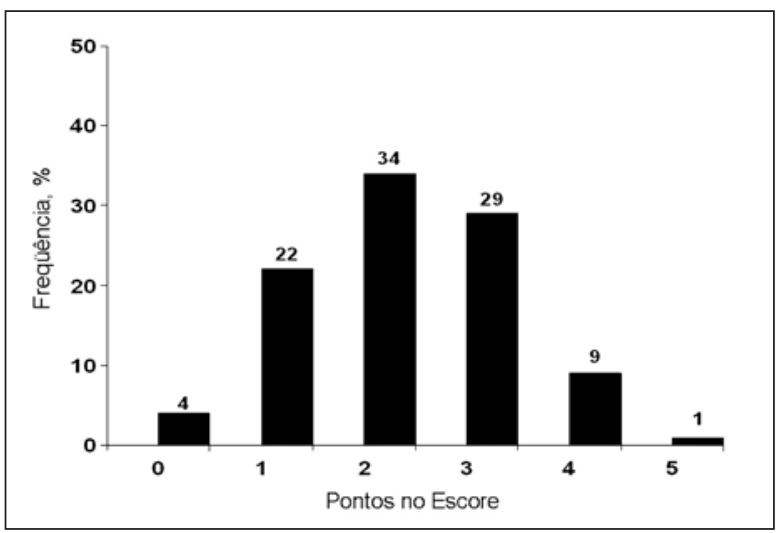

Figura 2 - Distribuição da freqüência dos pacientes analisados no presente estudo de acordo com os pontos no escore de risco.

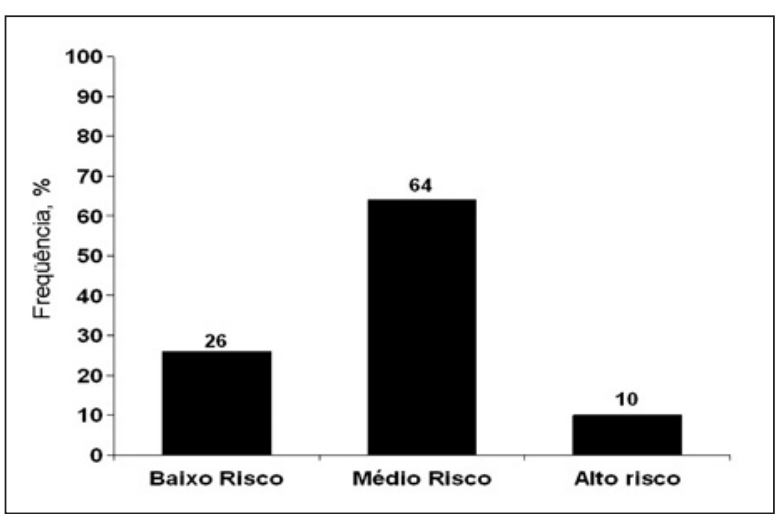

Figura 3 - Distribuição da freqüência dos pacientes analisados no presente estudo de acordo com as categorias de risco. ário para novas RVA, e somente $10 \%$ dos procedimentos foram realizados em indivíduos com alto risco quando avaliado pela ferramenta utilizada. Essa informação tem importância dentro da perspectiva da adoção do uso seletivo de stents farmacológicos pelo sistema público de saúde, já que essa estratégia representaria seu uso em não mais que $20 \%$ dos pacientes tratados atualmente.

Embora os stents farmacológicos sejam usados atualmente em cerca de $60 \%$ a $80 \%$ dos procedimentos realizados em alguns países desenvolvidos, análises de custo-efetividade considerando a perspectiva dos sistemas de saúde brasileiros não sugerem que essa seja atualmente uma estratégia economicamente viável no Brasil ${ }^{16}$. Assim, a estimativa do risco de novas RVA em populações tratadas contemporaneamente em nosso meio adquire importância para estimar seu potencial emprego na prática clínica diária caso venha a ser adotada. Como o principal benefício clínico dos stents farmacológicos consiste na diminuição da reestenose e de novos procedimentos de revascularização, seu uso preferencialmente naqueles pacientes propensos a esses desfechos parece ser a alternativa mais adequada dentro desse contexto, o que também foi demonstrado em alguns estudos recentes ${ }^{8,17}$.

Em nossos estudos prévios, os pacientes com alto risco de reestenose pelo escore utilizado apresentaram risco de novas revascularizações de $20 \%$ ou mais, e aqueles com baixo risco tiveram taxas de novas revascularizações de aproximadamente $5 \%{ }^{13,14}$. Enquanto esses parecem ser limites bastante razoáveis para indicar ou não stents farmacológicos, dois terços dos pacientes incluídos no presente estudo encontravamse em um perfil de risco intermediário, em que as taxas de revascularização estão estimadas entre $10 \%$ e $20 \%$, conforme demonstrado na Figura 1. A decisão do uso de stents farmacológicos nesses pacientes deve provavelmente ser individualizada, considerando outros fatores como envolvimento proximal da artéria descendente anterior, presença de reestenose prévia e potencial aderência aos antiplaquetários.

A capacidade de modelos estatísticos para predizer a reestenose e novas revascularizações após implante de stents coronarianos tem sido objeto de controvérsia. Em nossos estudos prévios para validação e desenvolvimento do escore utilizado, observamos associação altamente significativa entre este e as taxas de novas revascularizações, sugerindo ser possível a estimativa de pacientes com baixo e alto riscos ${ }^{13,14}$. Esses achados são concordantes com o modelo estatístico sugerido no estudo de Greenberg et al. ${ }^{12}$. Por outro lado, Singh et al. ${ }^{18}$ não identificaram boa capacidade preditiva de um escore de risco desenvolvido na população de pacientes incluída no ensaio clínico PRESTO. Da mesma forma, Ellis et al. ${ }^{19}$ analisaram a evolução clínica de aproximadamente 5.000 pacientes tratados com stents convencionais, concluindo pela 
Quadros AS, et al. Perfil de Risco de Reestenose em Pacientes Submetidos a Implante de Stents Coronarianos Convencionais. Rev Bras Cardiol Invas. 2008;16(2):155-159.

dificuldade em definir aqueles com muito baixo risco. Por outro lado, vários subgrupos de baixo risco identificados foram muito semelhantes àqueles encontrados em nossos estudos.

Um achado interessante do presente estudo foi que o perfil de risco de novas revascularizações da população estudada conforme o escore utilizado apresentou distribuição próxima da distribuição normal, com a maioria dos pacientes concentrando-se nos escores intermediários e poucos nos extremos. Até onde alcança nosso conhecimento, esse dado não havia sido previamente relatado, sendo poucos os estudos sobre o perfil de risco de reestenose de pacientes tratados com stents coronarianos. Em um estudo realizado com pacientes tratados com angioplastia coronariana, Rensing et al. ${ }^{20}$ analisaram a distribuição da perda tardia avaliada por angiografia quantitativa em aproximadamente 1.400 lesões. Esses autores demonstraram que a reestenose pode ser vista como o final da cauda de um fenômeno com distribuição quase normal, o que seria compatível com os resultados de nosso estudo.

De acordo com dados recentemente relatados provenientes do registro $\mathrm{CENIC}^{21}$, a utilização dos stents farmacológicos no Brasil tem crescido nos últimos anos, bem como as taxas de sucesso e a diminuição de complicações desses procedimentos. A expectativa é de que a ampliação do uso dessa tecnologia continue para um número cada vez maior de pacientes, mas as questões econômicas permanecem como uma limitação importante. Os resultados principais desse estudo, de que a minoria dos pacientes tratados contemporaneamente com stents coronarianos apresenta alto risco de reestenose, podem fornecer um balizamento importante para os órgãos regulatórios, no sentido de que essa importante tecnologia possa ser aprovada para aqueles pacientes com melhor relação custo-benefício.

\section{REFERÊNCIAS BIBLIOGRÁFICAS}

1. Sousa JE, Costa MA, Abizaid AC, Rensing BJ, Abizaid AS, Tanajura LF, et al. Sustained supression of neointimal proliferation by sirolimus-eluting stents: one-year angiographic and intravascular ultrasound follow-up. Circulation. 2001;104 (17):2007-11

2. Moses JW, Leon MB, Popma JJ, Fitzgerald PJ, Holmes DR, O'Shaughnessy C, et al.; SIRIUS Investigators. Sirolimus-eluting stents versus standard stents in patients with stenosis in a native coronary artery. N Engl J Med. 2003;349(14):1315-23.

3. Stone GW, Ellis SG, Cox DA, Hermiller J, O'Shaughnessy C, Mann JT, et al; TAXUS-IV Investigators. A polymerbased, paclitaxel-eluting stent in patients with coronary artery disease. N Engl J Med. 2004;350(3):221-31.

4. Rinfret S, Grines CL, Cosgrove RS, Ho KK, Cox DA, Brodie BR, et al.; Stent-PAMI Investigators. Quality of life after balloon angioplasty or stenting for acute myocardial infarction. One-year results from the Stent-PAMI trial. J Am Coll Cardiol. 2001;38(6):1614-21.

5. Nayak AK, Kawamura A, Nesto RW, Davis G, Jarbeau J, Pyne CT, et al. Myocardial infarction as a presentation of clinical in-stent restenosis. Circ J. 2006;70(8):1026-9.
6. Walters DL, Harding SA, Walsh CR, Wong P, Pomerantsev E, Jang IK. Acute coronary syndrome is a common clinical presentation of in-stent restenosis. Am J Cardiol. 2002;89(5):491-4.

7. Stettler C, Wandel S, Allemann S, Kastrati A, Morice MC, Schömig A, et al. Outcomes associated with drug-eluting and bare-metal stents: a collaborative network meta-analysis. Lancet. 2007;370(9591):937-48.

8. Tu JV, Bowen J, Chiu M, Ko DT, Austin PC, He Y, et al. Effectiveness and safety of drug-eluting stents in Ontario. N Engl J Med. 2007;357(14):1393-402.

9. Daemen J, Wenaweser P, Tsuchida K, Abrecht L, Vaina S, Morger C, et al. Early and late coronary stent thrombosis of sirolimus-eluting and paclitaxel-eluting stents in routine clinical practice: data from a large two-institutional cohort study. Lancet. 2007;369(9562):667-78.

10. Airoldi F, Colombo A, Morici N, Latib A, Cosgrave J, Buellesfeld, et al. Incidence and predictors of drug-eluting stent thrombosis during and after discontinuation of thienopyridine treatment. Circulation. 2007;116(7):745-54.

11. Lemos PA, Serruys PW, Sousa JE. Drug-eluting stents: costs versus clinical benefit. Circulation. 2003;107(24):3003-7.

12. Greenberg D, Bakhai A, Cohen DJ. Can we afford to eliminate restenosis? Can we afford not to? J Am Coll Cardiol. 2004;43(4):513-8.

13. Gottschall CA, Quadros AS, Sarmento-Leite R. Predictive score for target vessel revascularization after bare metal coronary stenting. J Invasive Cardiol. 2006;18(1):22-6.

14. Quadros AS, Diemer F, Gottschall CA. Validation of a risk score for a new target vessel revascularization after stent implantation. J Am Coll Cardiol. 2008;51(Suppl A):A222.

15. Smith SC Jr, Feldman TE, Hirshfeld JW Jr, Jacobs AK, Kern MJ, King SB $3^{\text {rd }}$, et al. ACC/AHA/SCAI 2005 guideline update for percutaneous coronary intervention: a report of the American College of Cardiology/American Heart Association Task Force on Practice Guidelines (ACC/AHA/SCAI Writing Committee to Update 2001 Guidelines for Percutaneous Coronary Intervention). Circulation. 2006;113(7): e166-286.

16. Quadros AS, Gottschall CAM, Sarmento-Leite R. Custoefetividade dos stents revestidos com drogas em vasos de grande calibre. Rev Bras Cardiol Invas. 2006;14(3):306-13.

17. Garg P, Cohen DJ, Gaziano T, Mauri L. Balancing the risks of restenosis and stent thrombosis in bare-metal versus drug-eluting stents: results of a decision analytic model. Am Coll Cardiol. 2008;51(19):1844-53.

18. Singh M, Gersh BJ, McClelland RL, Ho KK, Willerson JT, Penny WF, et al. Clinical and angiographic predictors of restenosis after percutaneous coronary intervention: insights from the Prevention of Restenosis With Tranilast and Its Outcomes (PRESTO) trial. Circulation. 2004;109(22):2727-31.

19. Ellis SG, Bajzer CT, Bhatt DL, Brener SJ, Whitlow PL, Lincoff AM, et al. Real-world bare metal stenting: identification of patients at low or very low risk of 9-month coronary revascularization. Catheter and Cardiovasc Interv. 2004;63(2):135-40

20. Rensing BJ, Hermans WR, Deckers JW, de Feyter PJ, Tijssen JG, Serruys PW. Lumen narrowing after percutaneous transluminal coronary balloon angioplasty follows a near gaussian distribution: a quantitative angiographic study in 1,445 successfully dilated lesions. J Am Coll Cardiol. 1992;19(5): 939-45.

21. Cardoso CO, Quadros AS, Mattos LA, Gottschall CA, Sarmento-Leite RE, Marin-Neto JA. Perfil de uso dos stents farmacológicos no Brasil: dados da Central Nacional de Intervenções Cardiovasculares (CENIC). Arq Bras Cardiol. 2007;89(6):356-61. 